Revue d'histoire de l'Amérique française

BEVUE D.HISTOIRE DE L'AMÉRIQUE FRANÇAISE

\title{
CHENNELLS, David, The Politics of Nationalism in Canada. Cultural Conflict Since 1760 (Toronto, University of Toronto Press, 2001), x-381 p.
}

\section{Steven Watt}

Volume 56, numéro 1, été 2002

URI : https://id.erudit.org/iderudit/007219ar

DOI : https://doi.org/10.7202/007219ar

Aller au sommaire du numéro

Éditeur(s)

Institut d'histoire de l'Amérique française

ISSN

0035-2357 (imprimé)

1492-1383 (numérique)

Découvrir la revue

Citer ce compte rendu

Watt, S. (2002). Compte rendu de [CHENNELLS, David, The Politics of Nationalism in Canada. Cultural Conflict Since 1760 (Toronto, University of Toronto Press, 2001), x-381 p.] Revue d'histoire de l'Amérique française, 56(1), 69-72. https://doi.org/10.7202/007219ar d'utilisation que vous pouvez consulter en ligne.

https://apropos.erudit.org/fr/usagers/politique-dutilisation/ 
«Jim Crow» dans leurs États, ont insisté pour que ceux-ci exercent le contrôle sur les régimes d'assurance-chômage. Tout cela dans le but de s'assurer que les Noirs sans emploi ne touchent pas de prestations.

ALVIN FINKEL

Centre des études de l'État et de la loi

Université Athabasca

CHENNELLS, David, The Politics of Nationalism in Canada. Cultural Conflict Since 1760 (Toronto, University of Toronto Press, 2001), x-381 p.

Ce livre de David Chennells est à la fois plus et moins ambitieux que son titre pourrait le laisser croire. Bien que disant traiter des politiques du nationalisme au Canada, l'ouvrage s'intéresse principalement à la question du nationalisme au Québec. Toutefois, des incursions au Manitoba et dans les Maritimes montrent que Chennells ne se limite pas au nationalisme franco-québécois. Et dans l'étude qu'il fait de l'Union, il s'attarde également aux manifestations du nationalisme britannique au Québec. Par ailleurs, l'auteur semble oublier que la discussion du nationalisme s'insère dans le récit plus général de l'histoire politique canadienne depuis la Conquête. Cette combinaison d'ambition et de modestie méthodologique donne un ouvrage souvent impressionnant dans ses parties, mais décevant dans son ensemble.

La partie la plus captivante de l'ouvrage demeure la longue discussion théorique présentée en introduction. L'auteur y discute les différents concepts de nationalisme avant de définir celui qu'il entend adopter. Pour lui, le nationalisme relève des buts d'un mouvement politique avant même de refléter sa composition ou encore la manière dont il est perçu. Chennells précise d'emblée qu'il considère le nationalisme comme un phénomène néfaste, du moins selon la perspective libérale qu'il adopte. Il s'intéresse particulièrement au "nationalisme exclusif officiel», dans lequel s'affirme la volonté de privilégier — voire d'imposer - un mode de vie particulier à l'ensemble d'une population en se servant du pouvoir de l'État. Pour sa part, Chennells emprunte le modèle théorique de conflict regulation, qui confie à l'État et aux autorités gouvernementales la prise en charge des conflits qui risquent de surgir au sein des populations qu'ils gouvernent. Ainsi, dans le cas du nationalisme, l'État doit rester a-national, ou du moins fournir un contexte où les élites de différentes origines peuvent surmonter les divisions 
nationales qui excitent le peuple. Les héros de l'histoire, pour l'auteur, sont donc ceux parmi les élites qui résistent aux influences nationalistes, que ce soit le gouverneur Carleton défendant les droits des Canadiens français contre les marchands britanniques, ou encore René Lévesque protégeant ceux des minorités linguistiques au Québec contre les nationalistes radicaux du mouvement souverainiste.

Les cinq chapitres qui forment le corps du livre traitent de périodes particulières de l'histoire canadienne au cours desquelles différents incidents sont étudiés dans le but de démontrer la capacité variable des gouvernements de contrôler les pulsions nationalistes des masses. À la suite de la Conquête, c'est l'ère de l'«imposed statescraft», qui implique la gestion coloniale par des gouverneurs jouissant d'une grande indépendance. Cette ère prend fin avec la violence des rébellions et leurs suites. L'union anglificatrice laisse sa place à l'«affiliative trusteeship", où les élites canadiennes de différentes origines se montrent souvent capables de s'entendre tout en maintenant une certaine indépendance vis-à-vis des électeurs. Cette tendance est surtout perceptible au sein du gouvernement central. L'avant-dernier chapitre retrace le développement du «nationalisme exclusif officiel » québécois au $\mathrm{xx}^{\mathrm{e}}$ siècle, période durant laquelle les législateurs québécois perdent leur indépendance et deviennent essentiellement des représentants de groupes ethniques particuliers. Enfin, l'échec des multiples tentatives de réconciliation constitutionnelle qui se sont succédé depuis les années 1970 démontrerait la difficulté d'en arriver à un accord entre les différents groupes culturels dans le contexte d'une importante participation populaire dans la vie politique.

En appliquant ainsi sa perspective théorique aux divers événements historiques, l'auteur offre un aperçu fort intéressant de plusieurs débats historiographiques, qu'il soit question de la Conquête, des Rébellions de 1837, de la Confédération, du duplessisme ou de la Révolution tranquille. Pourtant, le livre cherche aussi à tirer des conclusions générales sur l'histoire canadienne. Plus qu'une série de vignettes décrivant diverses tentatives de maîtriser les pulsions nationalistes, Chennells raconte l'histoire de la perte progressive de ce contrôle. Ainsi, la politique linguistique du gouvernement québécois des années 1960 et 1970 est présentée non seulement comme un exemple classique de "nationalisme exclusif officiel», mais aussi comme le résultat d'un long développement politique. Ce développement constituerait l'effet pervers de la libéralisation des institutions politiques. Ainsi, dans la mesure où le 
peuple exerce une influence directe sur les autorités politiques, celles-ci deviennent plus susceptibles de céder au nationalisme.

Bref, l'auteur oriente l'ensemble de l'histoire constitutionnelle canadienne autour d'un récit linéaire promouvant sa perspective théorique sur le nationalisme. Jamais il n'est question du flux et du reflux de l'importance de la (ou des) question(s) nationale(s). Il manque également une véritable mise en contexte du nationalisme qui tiendrait compte d'autres influences sur la vie politique. La démocratisation des institutions politiques, qui serait à l'origine de l'influence croissante du "nationalisme exclusif officiel», n'est pas expliquée ni examinée en détail. Et seule la conclusion aborde la véritable question morale qui se trouve au cour de l'ouvrage: la comparaison des effets néfastes du «nationalisme exclusif officiel» avec les autres changements apportés par la démocratisation des institutions politiques.

Globalement, l'auteur a tendance à tirer des conclusions générales à partir de preuves tout aussi générales. Par exemple, il suggère plusieurs raisons pour expliquer pourquoi les élites seraient moins attirées par le nationalisme que les masses (ce qu'il appelle "the power cleavage»): interdépendance des élites de différentes origines partageant des buts politiques, désir de stabilité intérieure face à des menaces extérieures, occasions plus fréquentes de voyager, horizons culturels plus larges, etc. Les preuves sont beaucoup moins nombreuses du côté du peuple: références sporadiques à des émeutes ou à des manifestations, à des sondages. Et jamais l'intérêt des élites pour le nationalisme ni les mouvements interethniques ou internationalistes parmi le peuple (il existe des exemples fort intéressants des deux phénomènes) ne sont analysés en détail. Par ailleurs, la définition d'élite employée est plutôt élastique. Elle peut exclure, au besoin, les élites locales, les élites moins conciliatrices et les élites en herbe.

En outre, le nationalisme au Canada est analysé presque uniquement à travers les relations établies entre les Canadiens anglais et les Canadiens français. Le rôle des autres groupes et la prise en compte des autres nationalismes sont absents du récit, même quand ils touchent directement au développement des nationalismes discutés. Par exemple, il n'est jamais question de relations entre Européens et Autochtones, ni même de la nouvelle vague de nationalisme autochtone et les relations souvent conflictuelles des groupes autochtones avec les gouvernements - notamment le gouvernement québécois - durant les dernières décennies. Quant aux autres facteurs qui pourraient influencer 
l'importance ou le visage du nationalisme, ils sont systématiquement mis de côté. Par exemple, un mépris des élites, souvent présent chez la population canadienne, est mentionné à plusieurs reprises pour expliquer l'attitude hostile de la population envers les projets conciliateurs de leurs représentants politiques. Cette contre-explication reste cependant au niveau de la parenthèse. Elle n'est jamais directement confrontée au nationalisme, afin d'évaluer ses influences respectives (et mutuelles) dans le contexte du développement politique général. Certes, dire que la réalité est plus complexe que la théorie relève autant du cliché que dire que le peuple est plus enclin au chauvinisme national que les élites. De toute façon, la théorie de Chennells est loin d'être simplificatrice. Le problème est autre: Chennells néglige de confronter sa théorie à l'empirique. Dès la deuxième phrase de l'introduction, il reconnaît que son but est de proposer une "theory of nationalism by considering Canadian history", mais pas l'inverse. Le livre de Chennells montre que cette approche permet d'offrir des aperçus intéressants sur divers événements isolés. Mais en faisant du nationalisme la clé de l'histoire politique canadienne et en négligeant d'autres pistes d'explication - même celles qui pourraient être mises en relation avec sa propre piste — l'auteur construit son récit global sur des fondements précaires.

STEVEN WATT

Département d'histoire

Université du Québec à Montréal

DAWSON, Nelson-Martin, L'Atelier Delisle. L'Amérique du Nord sur la table à dessin (Sillery, Septentrion, 2000), 306 p.

La production cartographique française de l'Amérique du Nord des $\mathrm{XVII}^{\mathrm{e}}$ et $\mathrm{XVIII}^{\mathrm{e}}$ siècles est une activité d'ateliers. Celui de Claude Delisle (1644-1729), situé dans la rue des Canettes, puis celui de Guillaume (1675-1726), fils aîné de Claude, au quai de l'Horloge, font de Paris un centre principal de la fabrication de cartes. Les coordonnées géographiques disponibles et vérifiées ainsi que les données extraites des récits des explorateurs et des voyageurs de l'Amérique du Nord, étalées sur les tables à dessin de l'atelier Delisle, aboutiront, en 1703, sur une carte de l'Amérique septentrionale qui est la meilleure représentation cartographique du territoire québécois du $\mathrm{XvIII}^{\mathrm{e}}$ siècle; puis, en 1718 , elles contribueront à la cartographie d'une vaste Louisiane. 